\title{
Some observations of a stationary and revolving three-dimensional skeletal cube ${ }^{1}$
}

\author{
HERMAN H. SPITZ ${ }^{2}$ \\ EDWARD R. JOHNSTONE TRAINING AND RESEARCH CENTER
}

\begin{abstract}
Observations were made of the reversal rate of a three-dimensional skeletal cube as a function of obsenving condition and state of rest or motion of the cube. Perspective reversal rate of the stationary three-dimensional cube is greater than direction reversal rate of the same cube set in motion. Reversal rate of the rotating cube drops with rest, replicating a similar finding with the Necker cube. Comparisons are made with the rotating trapezoidal window illusion.
\end{abstract}

Ian Howard (1961) has called attention to an interesting illusion. If one observes a three-dimensional skeletal cube rotating slowly in one direction, after a time the cube will suddenly appear to change the direction of its rotation. Howard was interested primarily in the latency of the first reversal under particular conditions, and in comparing the effects of monocular and binocular observation. Based on his results, he attributes this illusion to a neuronal, rather than an electrolytic, type of satiation. That is, patterns of movement are coded into spatially specific sequences, and these fixed spatial patterns become satiated. The satiation process is "specific to the particular rotation-in-depth of the stimulus figure [p. 27]."

It is possible that this particular illusion is induced by the same process underlying Necker cube reversals. We have repeatedly found that when, under neutral instructions, naive Ss observe the Necker cube steadily for $2 \mathrm{~min}$, the reversal rate describes a negatively accelerated function (Spitz \& Lipman, 1962). Furthermore, if this observation period is followed immediately by a 2 -min rest, the reversal rate drops approximately $50 \%$ of the way back to the starting level.

One purpose of the present experiment is to see whether or not the rate of direction reversal of the revolving cube also builds up over time and declines with rest. Additionally, the rate of perspective reversals when the cube is stationary will be compared with the rate of direction reversals when the cube is revolving.

\section{METHOD}

A 4-sq-in. skeletal cube was constructed of 1/16-in. balsa wood, painted white. It was tilted back at a 30-deg angle and supported at the center of one of its struts by a black $1 / 8$-in. dowel. When turned slightly, it resembled a Necker cube. ${ }^{3}$ It was set in this position to serve as a stationary cube, and it started its rotation from this identical position when observed as a revolving cube. It was housed in a black, lightproof box, $4 \mathrm{ft}$ from the viewing eyepiece. Lighting was provided by four hidden 3-W bulbs. Two bulbs were on the floor of the apparatus, one directly in front, and one directly behind the supporting dowel. Two were similarly placed on the ceiling, thereby eliminating wall shadows. The supporting dowel extended upward from a reversible 2-rpm motor, set for counterclockwise rotation. The fixation point, used for all but one group, was a $3 / 8$-in. brown tack placed $41 / 2$ in. behind the center of the cube.

To start a trial, E pressed a lever that simultaneously activated the lights, a .001 -min timer, and the motor (for groups observing the cube in motion). Another lever, situated 10 in. below the center of the eyepiece, was pressed by $S$ at each reversal. The S's initial lever press stopped the timer, providing a measure of latency. In addition, each lever press registered audibly on a counter.

The Ss, 138 volunteer female college sophomores, were randomly placed into one of five groups. They were instructed to press the lever each time the cube changed direction, or-in the stationary groups-changed perspective. All Ss observed with their right eyes only.

Group 1, the straight massed group $(\mathrm{N}=30)$, observed the revolving cube continuously for $4 \mathrm{~min}$.

Group 2, the interpolated rest group $(N=25)$, observed the revolving cube for $2 \mathrm{~min}$, rested $2 \mathrm{~min}$, then observed it for 2 min more.

Group 3, the unwinding group $(\mathrm{N}=28)$, was the only group that actually changed the direction of the cube's rotation. They observed the cube continuously for $4 \mathrm{~min}$, under instructions to keep the cube going in the same counterclockwise direction in which they first saw it going. If it appeared to change directions, they could change it back again by pressing the lever. This actually did change the direction of rotation. For example, if $S$ perceived an illusory change to clockwise rotation, she reversed the cube into veridical clockwise direction, in the mistaken belief that she was maintaining the cube's initial direction.

Groups $4 \quad(\mathrm{~N}=30)$ and $5 \quad(\mathrm{~N}=25)$ observed the cube in a stationary position continuously for $4 \mathrm{~min}$, pressing the lever at each perspective reversal, as in a Necker cube experiment. The only difference between these groups was that Group 5 did not have a fixation point.

\section{RESULTS}

The results are shown in Fig. 1, where reversals are plotted over 30-sec trials. Note that for the straight massed group, which observed the revolving cube steadily for $4 \mathrm{~min}$, the reversal rate curve increases up to about 2 to $21 \% \mathrm{~min}$, at which point it is asymptotic. The lack of a clear negatively accelerated function over the first $2 \mathrm{~min}$ supports Howard's observation that satiation has difficulty overcoming the bias toward three-dimensionality. Apparently it has overcome this bias by Trial 2 , however, since from Trial 2 to asymptote the curve approximates a negatively accelerated function.

In the interpolated rest group, a 2 -min rest significantly $(p<.05)$ drops the reversal rate, indicating that the reversals ae not due primarily to learning, but rather to some type of fatigue or satiation that partially recovers with rest. A strict satiation theory (Köhler \& Wallach, 1944) explanation is not adequate, however, since Price (1968) has demonstrated that the reversal rate of the rotating skeletal cube is different for the veridical and illusory components. Duration of perceived veridical rotation decreases at a negatively accelerated rate, while duration of illusory rotation remains constant.

The unwinding group, which actually changed the cube's direction to compensate for illusional changes, reports significantly $(p<.05)$ more reversals than the straight massed group. This conflicts with Howard's finding, under binocular conditions, that objectively changing the cube's direction immediately after an illusory reversal will increase the latency of the next reversal. If such were the case, the unwinding group would have observed fewer, not more, reversals. It seems possible that the objective change of the cube's direction brought out cues to depth 


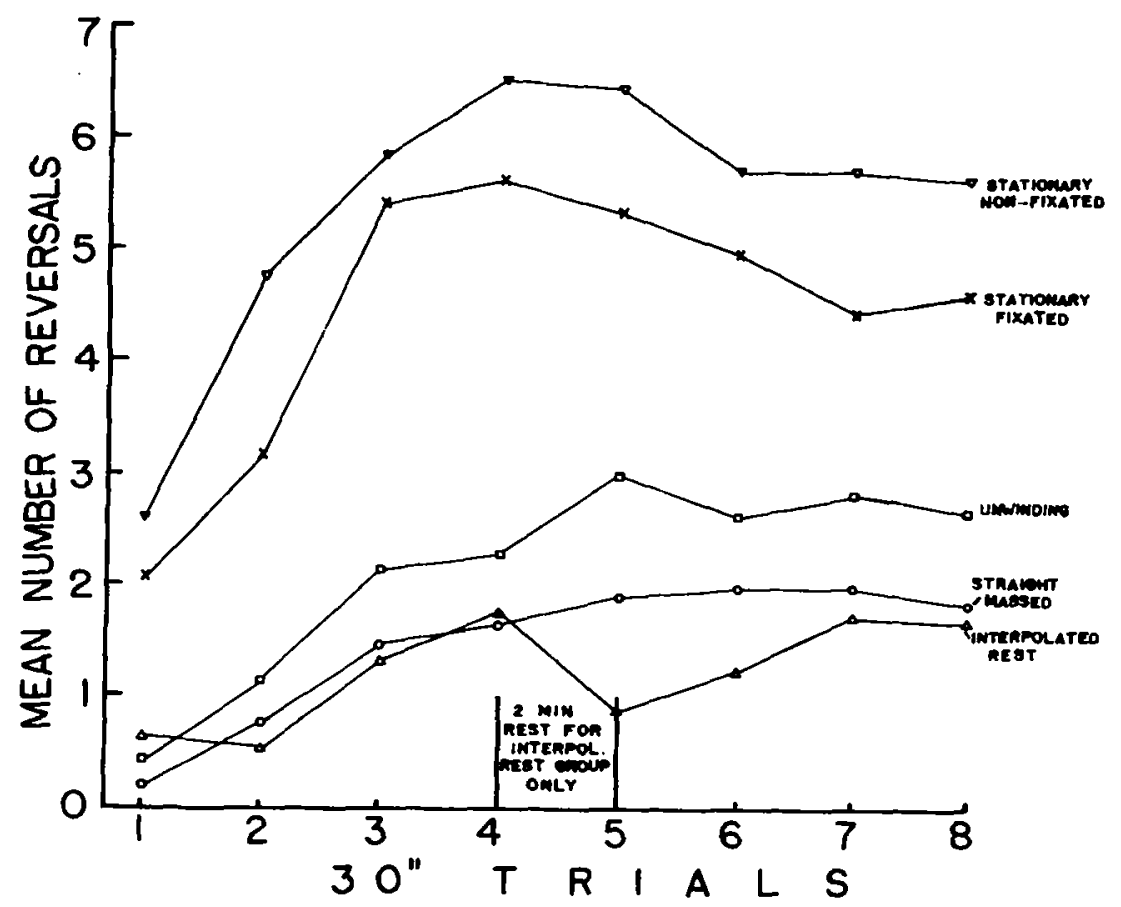

which periodically interrupted and overcame the effects of satiation, which in turn would be quickly reinstated. This interaction could result in the increased reversal rate.

The perspective reversal rate of the two stationary groups is far greater than the direction reversal rate reported by the three groups that observed the revolving cube. This supports satiation theory, since a stationary cube would excite the same cortical area more steadily and for a longer period of time than would a revolving cube (Howard, 1961). Additionally, if one invokes differential firing rates of directional cells (Hubel \& Wiesel, 1962), simultaneous opposing movements of the front and rear aspects of the cube over a large shared area may tend to cancel each other out, thus reducing the speed of directional satiation.

There are some interesting differences between the two stationary groups. The nonfixated group reports more total reversals, although the differences are not statistically significant. Over the first $2 \mathrm{~min}$, the curve of the nonfixated group is negatively accelerated from the first trial, while that of the fixated group is not. Both groups show a decline in reversal rate over the final $2 \mathrm{~min}$, but only for the fixated group is this decline reliable $(p<.05)$. It is possible that this decline is due to retinal fatigue or retinal fading of the cube outline, which would more readily occur under fixated conditions.
Fig. 1. Reversal rate of the five experimental groups as a function of observing condition and state of the three-dimensional cube (at reat or in motion).

conditions (slow speed, adequate distance or monocular viewing, painted shadows) oscillates much more predictably and steadily, essentially insensitive to observation time and rest. It appears that the oscillations of the classical trapezoidal window are primarily dependent on deception of the observer by manipulation of depth cues (Cross \& Cross, 1969; Spitz, 1964), while the cube reversals result primarily from continuing neural changes taking place in the observer from the moment of stimulation.

\section{REFERENCES}

ADAMS, P. A. The effect of past experience on the perspective reversal of a tridimensional figure. American Journal of Psychology, 1954, 67, 708-710.

Finally, there were no differences in latency of first response between the two stationary groups or between any of the three groups who observed the rotating cube. The latency of first response to the stationary cube was, of course, significantly shorter than to the rotating cube.

\section{DISCUSSION}

When the forward aspect of the cube is satiated, the rearward aspect, which is moving in the opposite direction, suddenly appears forward, and the cube seems to change direction. At this point, many Ss commented that the cube looked distorted. This distortion obviousiy occurs because the rearward aspect, actually farther away and subtending a smaller visual angle, now appears to be in front (Adams, 1954).

Some other intersting effects can be observed with this illusion. If a small object is attached to the cube, the object may appear to float in space, as in the rotating trapezoidal window illusion (Ames, 1951). Unlike the trapezoidal window illusion, however, it is possible to see the object float completely around the cube, as if it were a satellite. Placing a bar through the cube also produces some unusual effects. But the source of the cube illusion is surely not the same as the trapezoidal illusion. The cube illusion builds up over time and decreases with rest, and the point of reversal is unpredictable. The trapezoidal window, when presented under ideal
AMES, A. Visual perception and the rotating trapezoidal window. Psychological Monographs: General \& Applied, 1951, 65, No. 7.

CROSS, J., \& CROSS, J. The misperception of rotary motion. Perception \& Psychophysics, $1969,5,94-96$.

HOWARD, 1. P. An investigation of a satiation process in the reversible perspective of revolving skeletal shapes. Quarterly Joumal of Experimental Psychology, 1961, 13, 19-33.

HUBEL, D. H., \& WIESEL, T. N. Receptive fields, binocular interaction, and functional architecture in the cat's visual cortex. Joumal of Physiology, 1962, 160, 106-1 S4.

KÖHLER, W., \& WALLACH, H. Figural after-effects: An investigation of visual processes. Proceedings of the American Philosophical Society, 1944, 88, 269-357.

PRICE, J. R. Studies of reversible figures. Unpublished doctoral dissertation, University of Western Australia, 1968.

SPITZ, H. H. A comparison of mental retardates and normals on the rotating trapezoidal window illusion. Journal of Abnormal \& Social Psychology, 1964, 68, 574-578.

SPITZ, H. H., \& LIPMAN, R. S. Some factors affecting Necker cube reversal rate. Perceptual \& Motor Skills, 1962, 15, 611-625.

\section{NOTES}

1. The author is most grateful to Marshall P. Smith, Chairman of the Psychology Department of Trenton State College, for his aid in securing space and college students, and to David L. Hoats, of the Johnstone Research Department, for wiring the apparatus.

2. Address: Edward R. Johnstone Training and Research Center, Bordentown, New Jersey 08505.

3. In contrast, Howard's cube was supported at one of its corners and oriented in a diagonal axis.

(Accepted for publication August 27, 1969.) 